\title{
Determining the etiology of small bowel obstruction in patients without intraabdominal operative history: a retrospective study
}

\author{
Youngjin Jang, Sung Min Jung, Tae Gil Heo, Pyong Wha Choi, Jae Il Kim, Sung-Won Jung, \\ Heungman Jun, Yong Chan Shin, Eunhae Um \\ Department of Surgery, University of Inje College of Medicine, Ilsan Paik Hospital, Goyang, Korea
}

Purpose: Most of the causes of small bowel obstruction (SBO) in patients without a history of abdominal surgery are unclear at initial assessment. This study was conducted to identify the etiology and clinical characteristics of SBO in virgin abdomens and discuss the proper management.

Methods: A retrospective review involving operative cases of SBO from a single institute, which had no history of abdominal surgery, was conducted between January 2010 and December 2020. Clinical information, including radiological, operative, and pathologic findings, was investigated to determine the etiology of SBO.

Results: A total of 55 patients were included in this study, with a median age of 57 years and male sex (63.6\%) constituting the majority. The most frequently reported symptoms were abdominal pain and nausea or vomiting. Neoplasm as an underlying cause accounted for $34.5 \%$ of the cases, of which $25.5 \%$ were malignant cases. In patients aged $\geq 60$ years $(n=23)$, small bowel neoplasms were the underlying cause in 12 (52.2\%), of whom 9 (39.1\%) were malignant cases. Adhesions and Crohn disease were more frequent in patients aged $<60$ years. Coherence between preoperative computed tomography scans and intraoperative findings was found in $63.6 \%$ of the cases.

Conclusion: There were various causes of surgical cases of SBO in virgin abdomens. In older patients, hidden malignancy should be considered as a possible cause of SBO in a virgin abdomen. Patients with symptoms of recurrent bowel obstruction who have no history of prior abdominal surgery require thorough medical history and close follow-up.

Keywords: Ileus; Intestinal obstruction; Neoplasms; Abdominal pain; Etiology

\section{INTRODUCTION}

Small bowel obstruction (SBO) occurs in approximately $4.6 \%$ of patients after an intraabdominal surgery and gives rise to around 300,000 hospitalizations in a year in North America. Adhesions are the most prevalent cause of SBO, accounting for up to $70 \%$ of

Received: Aug 9, 2021 - Revised: Sep 23, 2021 - Accepted: Sep 23, 2021

Correspondence to: Sung Min Jung, M.D.

Department of Surgery, Ilsan Paik Hospital, Inje University College of

Medicine, 170 Juhwa-ro, Ilsanseo-gu, Goyang 10380, Korea

Tel: +82-31-911-7993, Fax: +82-31-911-7319

E-mail: sungmin@paik.ac.kr

ORCID: https://orcid.org/0000-0001-5346-7315

(C) 2022 The Korean Society of Coloproctology

This is an open-access article distributed under the terms of the Creative Commons Attribution NonCommercial License (https://creativecommons.org/licenses/by-nc/4.0) which permits unrestricted non-

commercial use, distribution, and reproduction in any medium, provided the original work is properly cited. cases $[1,2]$. There have been many discussions about SBO due to postoperative adhesions, for which nonoperative management has become the primary approach in patients with history of abdominal surgery $[3,4]$.

In patients with a "virgin abdomen," i.e., patients with no prior intraperitoneal surgery or procedures, abdominal wall hernia was the most frequent cause of SBO. Incarcerated hernias can occur in the inguinal, femoral, ventral, or umbilical regions, and can be noted on initial physical examinations [4]. Early surgical intervention for $\mathrm{SBO}$ has been the classic dogma when no hernia is detected in the virgin abdomen. These patients are more likely to have hidden malignancies; otherwise, they may have variant lesions that require prompt diagnosis and surgical treatment $[4,5]$.

On the other hand, a number of retrospective studies have proposed that most SBOs in the virgin abdomen more commonly have benign causes. They reported that $75 \%$ to $82 \%$ of patients 
could have been treated without surgery and avoided operative complications [6, 7]. Furthermore, radiological diagnosis has become more efficient in discovering malignant lesions due to technical advancement $[8,9]$.

A reliable method to manage $\mathrm{SBO}$ in a virgin abdomen has not formed a consensus because there is still limited proof of its etiology. Understanding the etiology of SBO in a virgin abdomen is important to decide on the appropriate surgical treatment and uncover the origin of obstruction. Thus, we focused on SBO cases, the causes of which at initial assessment are ambiguous due to the absence of any abdominal surgical history.

The purpose of this study was to determine the etiology of SBO in virgin abdomens and the clinical characteristics of these patients. This knowledge can be useful in predicting the cause of an $\mathrm{SBO}$ and developing an adequate, individualized management plan for each patient.

\section{METHODS}

A review of the operative cases of SBO without any history of abdominal surgery in a single institute (Ilsan Paik Hospital, Inje University College of Medicine, Korea), from January 2010 to December 2020 was performed retrospectively. Since the goal of this study was to determine the etiology of SBO in virgin abdomens, we only included patients who underwent surgical treatment for SBO and had the final diagnosis confirmed either through pathological or operative findings. The study was approved by the Institutional Review Board of Ilsan Paik Hospital (No. ISPAIK 202012-015) with a waiver for informed consent.

The surgical list that was managed by our general surgery department was used to identify patients who underwent surgery with SBO. Text search was performed on the list to identify the diagnosis and names of procedures that are relevant to the etiology of SBO. After identifying the operative cases for SBO, we excluded cases in which the causes of obstruction appeared to be obvious on preoperative imaging or physical examinations, such as postoperative adhesions, incarcerated abdominal wall hernia, and prediagnosed inflammatory disease. Patients were excluded if they underwent prior abdominal surgery, including appendectomy, cholecystectomy, cesarean section, and any laparotomy procedure performed for benign and malignant lesions. Patients without obstructive symptoms or evidence of bowel obstruction on imaging tests were also excluded. Bowel obstructive symptoms were defined as abdominal pain with nausea or vomiting, abdominal distension, and constipation. Imaging findings of SBO included obvious dilatation of the proximal small intestine, collapsed part distal to the obstructive lesion, prominent mucosal folds of the small intestine (valvulae conniventes), and air-fluid levels [10]. Other exclusion criteria were presence of small bowel neoplasms that were incidentally diagnosed without any bowel obstructive symptoms or signs of ileus on imaging studies, definitive peritoneal metastasis from other organs, paralytic ileus with- out any obstructive lesion, and SBO secondary to obvious colorectal malignancies. Patients who improved without surgical treatment and were not readmitted to the hospital due to recurrence of obstructive symptoms were also excluded from this study. In addition, those younger than 18 years were excluded from this study because the etiology of SBO in children was different from that in adults; most of the operative cases of SBO in children were due to congenital anomalies, intussusception, and incarcerated abdominal wall hernia.

Clinical characteristics, preoperative clinical course, laboratory, radiological, operative, and pathological findings, and follow-up data were examined using electronic medical records to determine the etiology of SBO. Pathological and operative findings were used to define the etiology of SBO. Clinical characteristics included age, sex, type of visit, number of previous visits or admissions, symptoms (abdominal pain, nausea/vomiting, constipation, diarrhea, gastrointestinal bleeding, unintentional weight loss [loss of $>10 \%$ of body weight over 3 months], intermittent relief of pain, and fever), leukocyte count, initial vital signs, and medical and trauma history.

The etiologies of SBO were categorized as small bowel neoplasms (benign and malignant), adhesions, stricture or inflammation, intussusceptions, foreign bodies, internal hernia, mesenteric volvulus, and other special causes. If there were concomitant causes, we categorized it as the primary cause of the lesion. For example, internal hernia due to peritoneal adhesions, and intussusception due to a small bowel tumor were categorized as adhesions and small bowel tumors, respectively.

\section{Statistical analysis}

Statistical analysis was performed using IBM SPSS Statistics for Windows, ver. 25.0 (IBM Corp., Armonk, NY, USA). Continuous variables were reported as mean \pm standard deviation. Differences between mean values were tested using the $t$-test. For analysis of categorical variables, the chi-square test or Fisher exact test were used. The differences observed were considered statistically significant with a $\mathrm{P}$-value of $<0.05$.

\section{RESULTS}

In a total of 55 patients, a greater number of male patients (63.6\%) were included than female patients, with an overall median age of 57 years (range, $18-89$ years). The most common presentations were abdominal pain (100\%) and nausea/vomiting (34.6\%). The clinical characteristics of the patients are summarized in Table 1.

The etiologies of SBO in the study group are shown in Table 2. Small bowel neoplasms were the underlying cause in 19 patients (34.5\%) of which malignant neoplasms were found in 14 cases ( $25.5 \%$ of total, $73.7 \%$ of small bowel neoplasms). Other etiologies included adhesions $(n=11)$, stricture/abscess $(n=13)$, primary intussusception $(\mathrm{n}=3)$, Crohn disease $(\mathrm{n}=3)$, foreign body $(n=3)$, internal hernia $(n=3)$, mesenteric volvulus $(n=1)$, and 
Table 1. Comparison of clinical information in different age subgroups

\begin{tabular}{|c|c|c|c|c|}
\hline Variable & $\begin{array}{c}\text { Total } \\
(n=55)\end{array}$ & $\begin{array}{l}\text { Old aged group } \\
(n=23)\end{array}$ & $\begin{array}{l}\text { Young aged group } \\
\qquad(n=32)\end{array}$ & $\begin{array}{c}\text { P-value } \\
\text { (old vs. young age group) }\end{array}$ \\
\hline \multicolumn{5}{|l|}{ Patients information } \\
\hline No. of patients & 55 & 23 & 32 & \\
\hline Age (yr) & $54.8 \pm 19.0$ & $73.1 \pm 8.7$ & $41.7 \pm 12.3$ & $<0.001$ \\
\hline Male sex & $35(63.6)$ & $14(60.9)$ & $21(65.6)$ & 0.718 \\
\hline The first onset of SBO (day) & $85.4 \pm 269.2$ & $156.9 \pm 398.4$ & $34.0 \pm 84.1$ & 0.158 \\
\hline The latest onset of SBO (day) & $5.4 \pm 16.8$ & $7.4 \pm 24.7$ & $3.9 \pm 7.2$ & 0.442 \\
\hline Intermittent relief of obstruction & $25(45.5)$ & $13(40.6)$ & $17(53.1)$ & 0.803 \\
\hline No. of previous visits to the hospital due to SBO & $0.7 \pm 1.2$ & $0.7 \pm 1.4$ & $0.6 \pm 1.0$ & 0.729 \\
\hline No. of previous hospitalizations due to SBO & $0.2 \pm 0.6$ & $0.1 \pm 0.5$ & $0.2 \pm 0.6$ & 0.718 \\
\hline Admissions via ER & $44(80.0)$ & $5(21.7)$ & $6(18.8)$ & $>0.999^{\mathrm{a}}$ \\
\hline \multicolumn{5}{|l|}{ Symptoms and laboratory findings } \\
\hline Fever & $3(5.5)$ & $1(4.3)$ & $2(6.3)$ & $>0.999^{\mathrm{a}}$ \\
\hline Abdominal pain & $55(100)$ & $23(100)$ & $32(100)$ & $>0.999^{a}$ \\
\hline Nausea/vomiting & $19(34.5)$ & $9(39.1)$ & $10(31.3)$ & 0.544 \\
\hline Constipation & $8(14.5)$ & $6(26.1)$ & $2(6.3)$ & $0.057^{\mathrm{a}}$ \\
\hline Diarrhea & $8(14.5)$ & $5(21.7)$ & $3(9.4)$ & $0.257^{\mathrm{a}}$ \\
\hline Melena/hematochezia & $7(12.7)$ & $1(4.3)$ & $6(18.8)$ & $0.219^{a}$ \\
\hline Weight loss & $2(3.6)$ & $2(8.7)$ & $0(0)$ & $0.170^{\mathrm{a}}$ \\
\hline Serum WBC count $(/ \mu \mathrm{L})$ & $10,240.2 \pm 4,262.9$ & $10,206.1 \pm 4,298.6$ & $10,264.7 \pm 4,305.9$ & 0.960 \\
\hline \multicolumn{5}{|l|}{ History of patients } \\
\hline Trauma history & $7(12.7)$ & $2(8.7)$ & $5(15.6)$ & $0.686^{a}$ \\
\hline History of other malignancies & $3(5.5)$ & $3(13.0)$ & $0(0)$ & $0.068^{\mathrm{a}}$ \\
\hline Cardiovascular disease & $17(30.9)$ & $14(60.9)$ & $3(9.4)$ & $<0.010$ \\
\hline Diabetes mellitus & $8(14.6)$ & $5(21.7)$ & $3(9.4)$ & $0.257^{a}$ \\
\hline Neurovascular disease & $4(7.3)$ & $4(17.4)$ & $0(0)$ & $0.026^{\mathrm{a}}$ \\
\hline Duration of admission (day) & $14.0 \pm 9.8$ & $15.0 \pm 10.5$ & $13.3 \pm 9.4$ & 0.516 \\
\hline ICU admission & $5(9.1)$ & $1(4.3)$ & $4(12.5)$ & $0.387^{\mathrm{a}}$ \\
\hline Emergency surgery & $18(32.7)$ & $6(26.1)$ & $12(37.5)$ & 0.374 \\
\hline Malignancy & $14(25.5)$ & $9(39.1)$ & $5(15.6)$ & 0.048 \\
\hline \multicolumn{5}{|l|}{ Types of SBO lesion } \\
\hline Neoplasmb & $15(27.3)$ & $10(43.5)$ & $5(15.6)$ & 0.022 \\
\hline Tumor-like lesion $^{c}$ & $6(10.9)$ & $3(13.0)$ & $3(9.4)$ & $0.686^{\mathrm{a}}$ \\
\hline Benign condition & 34 (61.8) & $10(43.5)$ & $24(75.0)$ & 0.018 \\
\hline
\end{tabular}

Values are presented as number only, mean \pm standard deviation, or number (\%).

Differences between mean values were tested with the t-test. For analysis of categorical variables, the chi-square test or Fisher exact test was used. P<0.05 was considered significant.

Old aged group, $\geq 60$ years; young aged group, $<60$ years.

SBO, small bowel obstruction; ER, emergency room; WBC, white blood cell; ICU, intensive care unit.

aFisher exact test was used. ${ }^{b}$ Both benign and malignant masses that were associated with abnormal cell growth. 'Benign cyst or an inflammatory polyp.

heterotrophic pancreas $(n=2)$.

Table 2 shows the difference in etiologies between the 2 groups that were categorized based on patient age. In the older age group
( $\geq 60$ years, $n=23$ ), small bowel neoplasms were the most common, which was present in 12 cases (52.2\%), of which malignant cases were $9(39.1 \%)$. Adhesion $(\mathrm{n}=7,21.9 \%)$ and Crohn disease 


\section{Coloproctology romgin ange etal}

Table 2. Etiology of small bowel obstruction (SBO) in virgin abdomen

\begin{tabular}{lccc}
\hline Variable & Old aged group $(\mathrm{n}=23)$ & Young aged group $(\mathrm{n}=32)$ & Total $(\mathrm{n}=55)$ \\
\hline SB0 due to neoplasms & $12(52.2)^{\mathrm{a}}$ & $7(21.9)$ & $19(34.5)$ \\
$\quad$ Malignant & $9(39.1)^{\mathrm{a}}$ & $5(15.6)$ & $14(25.5)$ \\
Benign & $3(13.0)$ & $2(6.3)$ & $5(9.1)$ \\
Adhesions & $4(17.4)$ & $7(21.9)$ & $11(20)$ \\
Stricture/Inflammation/abscess & $2(8.7)$ & $11(34.4)$ & $3(23.6)$ \\
Crohn disease & $0(0)$ & $1(3.4)$ & $3(5.5)$ \\
Intestinal tuberculosis & $0(0)$ & $2(6.3)$ & $1(1.8)$ \\
Primary intussusception & $1(4.3)$ & $3(9.4)$ & $3(5.5)$ \\
Foreign body & $0(0)$ & $1(3.1)$ & $3(5.5)$ \\
Internal hernia & $2(8.7)$ & $1(3.1)$ & $3(5.5)$ \\
Heterotrophic pancreas & $1(4.3)$ & $0(0)$ & $2(3.6)$ \\
Mesenteric volvulus & $1(4.3)$ & $32(100)$ & $1(1.8)$ \\
Total & $23(100)$ & $55(100)$ & \\
\hline
\end{tabular}

Values are presented as number (\%).

Old aged group, $\geq 60$ years; young aged group, $<60$ years.

${ }^{a}$ One case of malignant tumors was SBO due to unknown descending colon cancer with omental invasion.

$(\mathrm{n}=3,9.4 \%)$ were more common in the younger age group $(<60$ years, $\mathrm{n}=32$ ). As shown in Table 1 , small bowel malignancy was more prevalent in the older age group than in the young age group $(n=9,39.1 \%$ vs. $n=5,15.6 \% ; P=0.048)$.

Table 3 shows the differences between patients with benign causes (benign group, $\mathrm{n}=41$ ) and malignant neoplasms (malignant group, $\mathrm{n}=14$ ). In the malignant group, constipation was more common than in the benign group, presenting in $35.7 \%$ and $7.3 \%$ respectively $(\mathrm{P}=0.02)$. In the benign group, patients tended to be hospitalized more often before surgery. The number of previous admissions was $0.17 \pm 0.59$ in the benign group $(\mathrm{P}=0.037)$. The rate of emergent surgeries was higher $(n=18,43.9 \%)$ in the benign group $(\mathrm{P}<0.01)$.

The mean age of 14 patients diagnosed with malignancies was $68.1 \pm 11.2$ years (data of patients diagnosed with malignancy-associated with SBO are shown in Supplementary Table 1) Of these patients, 3 had small bowel lymphomas, 1 had small bowel adenocarcinomas, 2 had small bowel neuroendocrine tumors, 2 had high-risk gastrointestinal stromal tumors (GISTs), and 5 had lowrisk GISTs. Small bowel GISTs with low risk were regarded as malignant lesions because of their malignancy potency. Three of the 5 patients, who were diagnosed with low-grade GISTs, had hematochezia or melena with intermittent abdominal cramping pain.

Ten patients were diagnosed with benign tumors and tumor-like lesions including, 2 benign cysts, an inflammatory polyp, an inflammatory fibroid tumor, and an ileal lipoma. Four cases were associated with small bowel intussusception due to a tumor.

Three patients were diagnosed with Crohn disease after surgery. One patient had a pelvic abscess with an ileal fistula due to Crohn disease. The other 2 patients had SBOs due to ileal strictures, whose diagnoses were confirmed pathologically. All the 3 patients underwent small bowel resection and anastomosis.

There were SBO cases related to a history of abdominal trauma. It was found that these patients had adhesions, strictures, and ischemic changes intraoperatively, which were considered to be caused by abdominal wall trauma. In our study, trauma history included in-car or out-car accidents, falls, and other crashing injuries that can cause blunt trauma to the abdominal wall. Six patients had a history of abdominal trauma (10.9\%), of whom 2 SBO patients had a history of car or bicycle accidents 1 or 2 months prior to their presentation of SBO symptoms. None of the patients had bowel obstructive symptoms immediately after the accident. On average, abdominal pain started and was exacerbated over 2 months after the accident.

Three patients had SBO caused by foreign bodies and underwent surgery. Among these patients, 1 had a $7 \mathrm{~cm}$-sized bezoar in the small bowel removed by an open enterotomy. Ileal fish bones were found in 2 other cases, with no presence of perforations or abscesses, which were removed by laparoscopy-assisted enterotomy with an incision of approximately $3 \mathrm{~cm}$.

Preoperative abdominal computed tomography (CT) scans were performed on all 55 patients in this study. CT diagnosis was compared with the final diagnosis that was confirmed by operative or pathological results. The results of abdominal CT diagnosis were congruent with the final diagnosis in $63.6 \%$ of patients (Table 4 ).

The majority of surgery (37 cases, 67.3\%) was small bowel resection and anastomosis. 17 cases were done by laparoscopic approach. The detailed types of surgery were shown in Supplementary Table 2. 
Table 3. Comparison of malignant and benign subgroups

\begin{tabular}{|c|c|c|c|}
\hline Variable & Malignant & Benign & P-value \\
\hline \multicolumn{4}{|l|}{ Patients information } \\
\hline No. of patients & 14 & 41 & \\
\hline Age (yr) & $68.1 \pm 11.6$ & $50.3 \pm 19.0$ & $<0.010$ \\
\hline Male sex & $9(64.3)$ & $26(63.4)$ & 0.953 \\
\hline The first onset of SBO (day) & $40.6 \pm 94.4$ & $100.7 \pm 306.6$ & 0.726 \\
\hline The latest onset of SBO (day) & $11.8 \pm 31.3$ & $3.1 \pm 6.4$ & 0.350 \\
\hline Intermittent relief of obstruction & $8(57.1)$ & $17(41.5)$ & 0.309 \\
\hline No. of previous visits to the hospital due to SBO & $1.0 \pm 1.4$ & $0.6 \pm 1.1$ & 0.987 \\
\hline No. of previous hospitalizations due to SBO & $0.1 \pm 0.5$ & $0.2 \pm 0.6$ & 0.037 \\
\hline Admissions via ER & $8(57.1)$ & $36(87.8)$ & $0.022^{\mathrm{a}}$ \\
\hline \multicolumn{4}{|l|}{ Symptoms and laboratory findings } \\
\hline Abdominal pain & $14(100)$ & $41(100)$ & $>0.999$ \\
\hline Nausea/vomiting & $4(28.6)$ & $15(36.6)$ & 0.749 \\
\hline Constipation & $5(35.7)$ & $3(7.3)$ & $0.020^{\mathrm{a}}$ \\
\hline Diarrhea & $0(0)$ & 8 (19.5) & $0.098^{\mathrm{a}}$ \\
\hline Melena/hematochezia & $4(28.6)$ & $3(7.3)$ & $0.061^{\mathrm{a}}$ \\
\hline Weight loss & $2(14.3)$ & $0(0)$ & $0.061^{\mathrm{a}}$ \\
\hline Serum WBC count $(/ \mu \mathrm{L})$ & $8,445.7 \pm 3,249.1$ & $10,852.9 \pm 4,426.1$ & 0.247 \\
\hline \multicolumn{4}{|l|}{ History of patients } \\
\hline Trauma history & $1(7.1)$ & $6(14.6)$ & $0.664^{\mathrm{a}}$ \\
\hline Other malignancy & $2(14.3)$ & $1(2.4)$ & $0.156^{\mathrm{a}}$ \\
\hline Cardiovascular disease & $9(64.3)$ & 8 (19.5) & $<0.010^{\mathrm{a}}$ \\
\hline Diabetes mellitus & $2(14.3)$ & $6(14.6)$ & $>0.999^{\mathrm{a}}$ \\
\hline Neurovascular disease & $3(21.4)$ & $1(2.4)$ & $0.047^{\mathrm{a}}$ \\
\hline Duration of admission (day) & $14.3 \pm 5.6$ & $13.9 \pm 11.0$ & 0.612 \\
\hline ICU admission & $1(7.1)$ & $4(9.8)$ & $>0.999^{\mathrm{a}}$ \\
\hline Emergency surgery & $0(0)$ & $18(43.9)$ & $<0.010^{\mathrm{a}}$ \\
\hline
\end{tabular}

Values are presented as number only, mean \pm standard deviation, or number (\%).

Differences between mean values were tested with the t-test. For analysis of categorical variables, the chi-square test or Fisher exact test was used. $\mathrm{P}<0.05$ was considered significant.

SBO, small bowel obstruction; ER, emergency room; WBC, white blood cell; ICU, intensive care unit.

aFisher exact test was used.

\section{DISCUSSION}

The presence of a hidden malignancy is taken into account when deciding on a surgical intervention to manage $\mathrm{SBO}$ in a virgin abdomen, especially if the cause of obstruction is unclear in the preoperative radiological findings. After investigating 55 patients with virgin abdomens, we found that small bowel neoplasm (36.4\%) was the most common cause of SBO, with malignant neoplasms accounting for $25.5 \%$ of the cases. Previous studies have reported various rates of malignancy in SBO patients with virgin abdomens, ranging from $3.0 \%$ to $37.5 \%$ [3]. One such study reported that a combined rate of benign and malignant neoplasms in the small bowel and mesentery accounted for $13 \%$ of SBO patients with virgin abdomens [6].

Several studies that have discussed the nonoperative treatment of SBO reported that the rate of surgical laparotomies showed no difference between patients with and without previous abdominal surgery [8,9]. Ng et al. [9] and Collom et al. [11] reported the rate of small bowel neoplasms as $3.0 \%$ to $4.2 \%$ and the most common cause of SBO as adhesions. Approximately $40 \%$ to $49 \%$ of patients with virgin abdomens are treated conservatively with gastrografin $[9,11]$.

Despite the lower rate of operative exploration for malignancies in virgin abdomens with $\mathrm{SBO}$, we found that the malignant rate 
Table 4. Diagnostic consistency of abdomen computed tomography (CT) scan

\begin{tabular}{|c|c|c|c|}
\hline \multirow{2}{*}{ Diagnosis } & \multirow{2}{*}{$\begin{array}{c}\text { No. of } \\
\text { diagnosis }\end{array}$} & \multicolumn{2}{|c|}{ CT diagnosis } \\
\hline & & Consistent & Inconsistent \\
\hline Total & 55 & $35(63.6)$ & $20(36.4)$ \\
\hline Small bowel neoplasm & 18 & 16 & 2 \\
\hline Malignant neoplasm & 6 & & \\
\hline Benign neoplasm & 10 & & \\
\hline Stricture & 1 & & \\
\hline Heterotrophic pancreas & 1 & & \\
\hline Stricture/abscess & 12 & 5 & 7 \\
\hline Stricture/abscess & 5 & & \\
\hline Crohn disease & 2 & & \\
\hline Tuberculosis enteritis & 1 & & \\
\hline Adhesion & 4 & & \\
\hline Malignant tumor & 2 & & \\
\hline Other malignancy & 1 & & \\
\hline Adhesion & 7 & 5 & 2 \\
\hline Adhesions & 5 & & \\
\hline Stricture & 1 & & \\
\hline Internal hernia & 1 & & \\
\hline Intussusception & 5 & 4 & 1 \\
\hline Intussusception & 3 & & \\
\hline Malignant tumor & 1 & & \\
\hline Stricture & 1 & & \\
\hline Internal hernia & 4 & 1 & 3 \\
\hline Internal hernia & 1 & & \\
\hline Stricture/abscess & 2 & & \\
\hline Adhesion & 1 & & \\
\hline Foreign body & 3 & 3 & 0 \\
\hline Mesenteric volvulus & 1 & 1 & 0 \\
\hline Paralytic ileus & 4 & 0 & 4 \\
\hline Stricture/inflammation & 2 & & \\
\hline Adhesion & 1 & & \\
\hline Heterotrophic pancreas & 1 & & \\
\hline Ischemic/thrombus & 1 & 0 & 1 \\
\hline Internal hernia & 1 & & \\
\hline
\end{tabular}

Values are presented as number only or number (\%).

was slightly higher than that reported in previous studies, which was most likely due to the inclusion of only those patients who underwent surgery for SBO. Moreover, we included more elderly patients, who had a higher rate of neoplasms and malignancy (52.2\% and $39.1 \%$, respectively). Considering these results, suspecting hidden malignancies should be of importance when eval- uating older age patients before deciding to conservatively treat the SBO in virgin abdomens. Even though SBOs in virgin abdomens are most likely to have a benign cause, it is worth noting that we should always be cautious of relying on the interpretation of radiological imaging when suspecting an underlying malignancy. If nonoperative treatment is favored, regular follow-ups and a detailed review of personal and family history should be carried out [8].

Benign lesions that caused SBO in this study included 2 benign cysts, an inflammatory polyp, an inflammatory fibroid tumor, and an ileal lipoma. Only preoperative CT findings revealed intussusception in 3 of these cases. Unlike in children, adult intussusceptions are frequently associated with neoplasms or other pathologic lesions. Azar and Berger [12] reported that $94 \%$ of adult intussusceptions were related to pathological lesions, and $48 \%$ of the intestinal lesions were malignant. In our study, 5 out of 40 (12.5\%) benign lesions and 2 of 15 (13.3\%) malignant neoplasms were associated with small bowel intussusception. Pathological, radiological, and operative findings of the patients diagnosed with malignancies in this study are shown in Supplementary Table 1. One patient with small bowel lymphoma showed ileocecal intussusception on abdominal CT. Another patient had mechanical ileus with jejunal obstruction due to descending colon cancer with omental invasion, which was initially thought to be jejunal tuberculosis.

All patients with SBO in this study experienced abdominal pain, out of which $45 \%$ had self-relieved intermittent abdominal pain. It was previously reported that the rate of obstruction in patients with small bowel malignancy as the first manifestation was $5 \%$ to $38 \%$ [13]. Although primary tumors have been responsible for $1.5 \%$ of SBOs, intermittent partial obstruction has been regarded as the most characteristic evidence of small bowel malignancies. Treatment of an unnoticed small bowel malignancy can be delayed for 4 to 9 months on an average while before it grows large enough to induce a mass effect and cause partial obstruction [14]. The incidence of SBO due to Crohn disease was reported to be 7\% to 16\% [14-16] and Miller et al. [14] found that Crohn disease was the second most common cause of SBO (7\%), followed by adhesions. Our study included 3 patients who were diagnosed with Crohn disease with a mean age of 33.7 years. One patient was a 21-year-old man who had an abrupt onset abdominal pain 1 hour before he came to the emergency room. His abdominal $\mathrm{CT}$ revealed $\mathrm{SBO}$ and jejunal intussusception and he underwent laparoscopy-assisted small bowel resection and anastomosis. Creeping fat signs were found intraoperatively (Fig. 1) and a focal ulcer with active ileitis was found on pathological testing, both of which made us suspect the diagnosis to be Crohn disease.

Small bowel injury after blunt abdominal trauma is rare, accounting for less than $1 \%$ of blunt trauma admissions [17]. Approximately $10.9 \%$ of SBO in virgin abdomens appeared to be induced by adhesions or ischemic injuries associated with abdominal wall trauma. Two out of 6 SBO patients had histories of ab- 

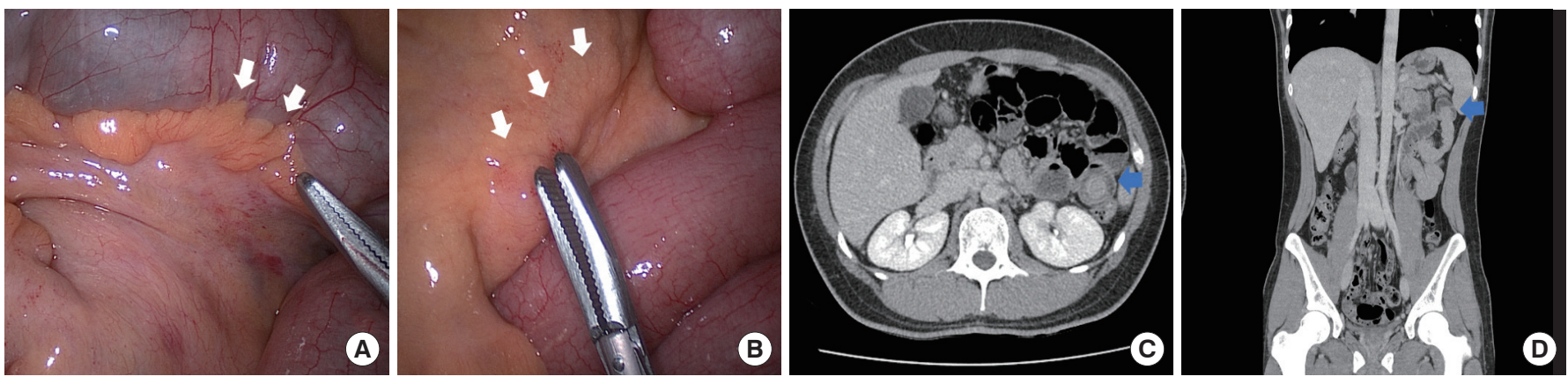

Fig. 1. The operative findings of a 21 years-old male patient with small bowel obstruction diagnosed with Crohn disease. (A) Creeping fat sign and (B) mesenteric thickening due to fibrofatty proliferation of the mesenteric tissue adjacent to chronic inflammation of bowel loops (white arrows). (C, D) The abdomen computed tomography scan shows targetoid small bowel (blue arrows) which is intussusception.
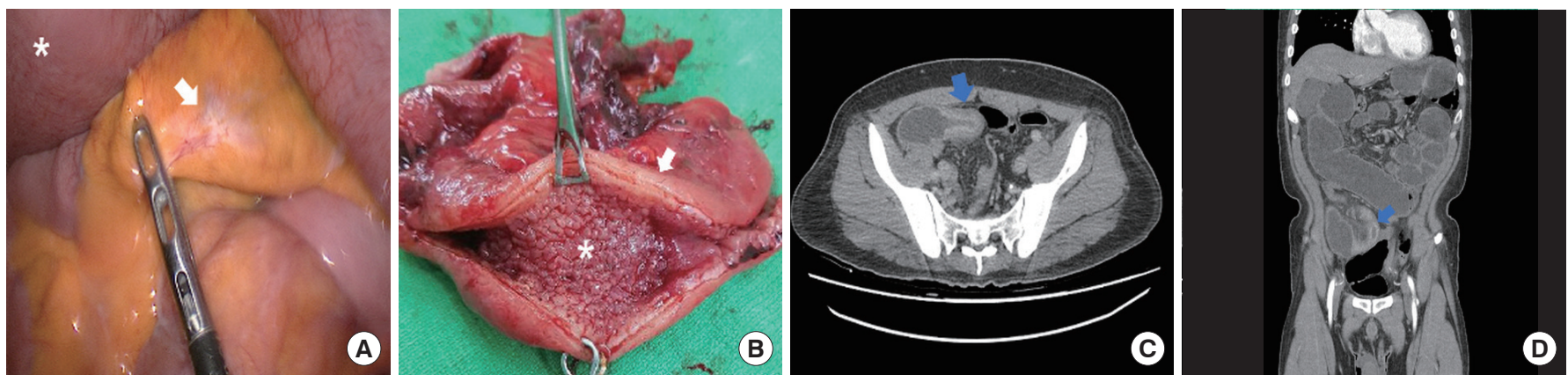

Fig. 2. The operative findings of a 48-year-old male patient who had small bowel obstruction after abdominal blunt trauma history. (A) The intraoperative finding. The fibrotic change in mesentery was caused by trauma (white arrows) and proximal bowel distension (asterisk). The distal part of the injured bowel was collapsed. (B) Gross finding of the specimen after the small bowel resection. Bowel wall thickening (white arrows) and segmental stenosis (asterisk) were associated with ischemic changes that were caused by a tear of the mesenteric vessel. (C, D) The abdomen computed tomography scan shows small bowel ileus and abrupt segmental stricture in small bowel adjacent to terminal ileum (blue arrows).
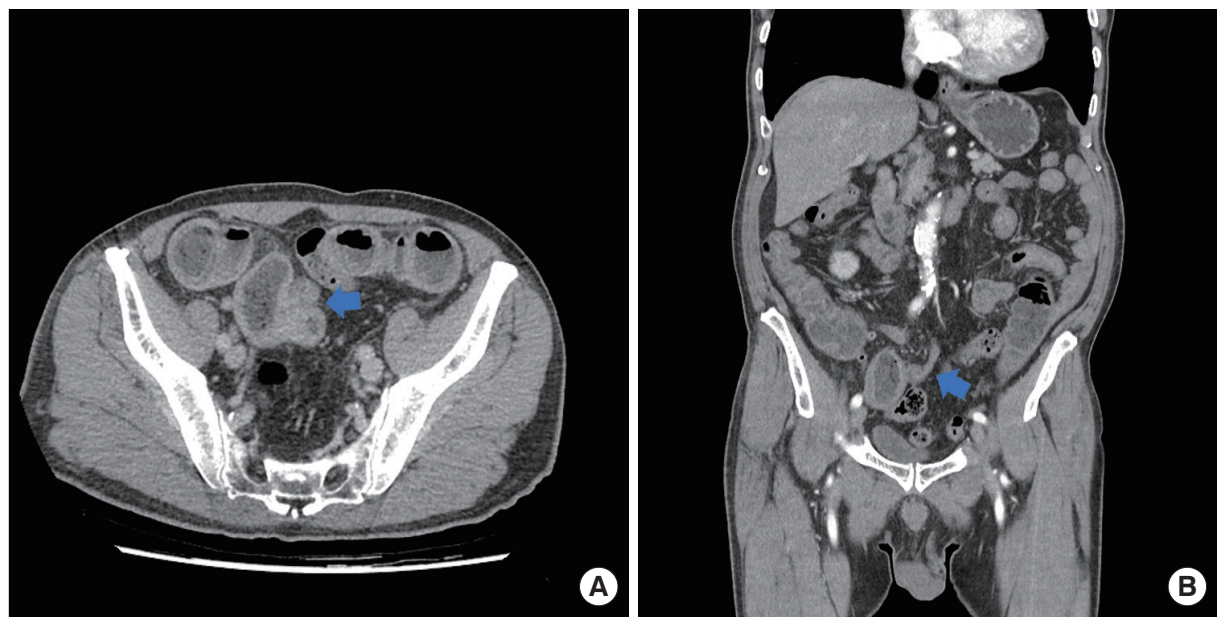

Fig. 3. The abdomen CT findings of an 80-year-old male patient who had recurrent SBO without any surgical history. Initial CT scan shows segmental stricture of small bowel and mild distension of proximal small intestine without mass-like lesions (blue arrows) in both axial veiw (A) and coronal veiw (B). This patient was finally diagnosed with small bowel adenocarcinoma postoperatively. 
dominal blunt trauma due to in-car accidents and had experienced intermittent abdominal pain for 2 months which worsened over time. An 80-year-old man, who had a history of falling from a persimmon tree during his childhood, had SBO with adhesions and internal hernia due to unknown causes. Another patient, who was a 48-year-old man, had a history of abdominal injury from a bicycle handle 2 months prior to experiencing SBO. Ischemic ileitis due to mesenteric vessel injury was diagnosed and the patient underwent laparoscopy-assisted small bowel resection and anastomosis. Intraoperative findings showed a segmental stricture of the ileum (Fig. 2).

Gallstone ileus is a rare etiology of SBO; only $1 \%-4 \%$ of gallstones may cause bowel obstruction. Most gallstone ileus occurs in the ileum (60.5\%) and can spontaneously resolve in $1.3 \%$ of cases. Obstruction in other intestinal organs is also possible in the jejunum (16.1\%), stomach (14.2\%), colon (4.1\%), and duodenum (3.5\%) [18].

Strajina et al. [8] reported an accuracy of 76\% (32 of 42) of CT scans in diagnosing SBO without prior abdominal surgery. Similarly, our study reported consistency of 63.6\% (35 of 55) of preoperative CT findings with the final diagnosis. Considering the occasional inaccuracy of CT scans, even when a CT scan does not show a mass-like lesion in the small bowel, the possibility of malignancy should still be considered. According to the results of this study, SBO followed by a lesion associated with stricture or intussusception and malignancy cannot be excluded. There was a case of an 80-year-old male patient who had recurrent SBO without any surgical history. His initial CT scan only showed short segmental stricture of small bowel and mild distension of proximal small intestine (Fig. 3). His symptoms were relieved several times after conservative treatment. This patient was finally diagnosed with small bowel adenocarcinoma postoperatively.

This study has several limitations. First, this was a single-center retrospective study with limited sample size. Although the cases in this series have been collected over 10 years, small bowel diseases, especially malignancies, are not common; hence, the sample size is limited. Moreover, we excluded all patients who had $\mathrm{SBO}$ and whose symptoms were relieved by conservative treatment alone to accurately determine the origin of small bowel pathology. Since we included only surgical cases, other treatment options for SBO in the virgin abdomen have not been discussed. In this study, we attempted to confirm the diagnosis and etiology of the SBOs of the patients. However, it was not possible to compare conservative and operative management of SBO in virgin abdomens. Further studies that are multi-centered, with a large number of patients and long-term follow-up data should be carried out to explore the etiology and management of SBO in virgin abdomens.

In conclusion, this review focuses on surgical cases of SBO in virgin abdomens, which are rarely studied in the literature. We found that neoplasms were the most common cause of SBO in patients with a virgin abdomen undergoing surgery, with a malig- nancy rate of $25.5 \%$. This result highlights the importance of suspecting neoplasms and malignancies as underlying causes in a patient without prior abdominal surgical history experiencing recurrent bowel obstructive symptoms from an unknown cause. It should also be noted that the possibility of malignancy is especially higher in older patients with SBO. In younger patients, benign etiologies, such as inflammatory bowel disease, are more common. Thus, thorough history taking may be important. If these patients are planned for conservative treatment instead of surgical treatment, close follow-up is recommended. Diagnostic laparoscopy should be considered instead of radiological imaging alone for recurrent bowel obstruction without a definite cause.

\section{CONFLICT OF INTEREST}

No potential conflict of interest relevant to this article was reported.

\section{FUNDING}

None.

\section{ORCID}

Youngjin Jang, https://orcid.org/0000-0001-9271-987X

Sung Min Jung, https://orcid.org/0000-0001-5346-7315

Tae Gil Heo, https://orcid.org/0000-0001-7057-4542

Pyong Wha Choi, https://orcid.org/0000-0001-5777-8436

Jae Il Kim, https://orcid.org/0000-0001-5984-2542

Sung-Won Jung, https://orcid.org/0000-0002-7537-4731

Heungman Jun, https://orcid.org/0000-0003-2530-4388

Yong Chan Shin, https://orcid.org/0000-0001-9808-1213

Eunhae Um, https://orcid.org/0000-0001-9688-7993

\section{SUPPLEMENTARY MATERIALS}

Supplementary materials for this study are presented online (available at https://doi.org/10.3393/ac.2021.00710.0101).

\section{REFERENCES}

1. Mullan CP, Siewert B, Eisenberg RL. Small bowel obstruction. AJR Am J Roentgenol 2012;198:W105-17.

2. Scott FI, Osterman MT, Mahmoud NN, Lewis JD. Secular trends in small-bowel obstruction and adhesiolysis in the United States: 1988-2007. Am J Surg 2012;204:315-20.

3. Choi J, Fisher AT, Mulaney B, Anand A, Carlos G, Stave CD, et al. Safety of foregoing operation for small bowel obstruction in the virgin abdomen: systematic review and meta-analysis. J Am Coll Surg 2020;231:368-75.

4. Dayton MT, Dempsey DT, Larson GM, Posner AR. New paradigms in the treatment of small bowel obstruction. Curr Probl 
Surg 2012;49:642-717.

5. McCloy C, Brown TC, Bolton JS, Bowen JC, Fuhrman GM. The etiology of intestinal obstruction in patients without prior laparotomy or hernia. Am Surg 1998;64:19-23.

6. Tavangari FR, Batech M, Collins JC, Tejirian T. Small bowel obstructions in a virgin abdomen: is an operation mandatory? Am Surg 2016;82:1038-42.

7. Beardsley C, Furtado R, Mosse C, Gananadha S, Fergusson J, Jeans $\mathrm{P}$, et al. Small bowel obstruction in the virgin abdomen: the need for a mandatory laparotomy explored. Am J Surg 2014;208:243-8.

8. Strajina V, Kim BD, Zielinski MD. Small bowel obstruction in a virgin abdomen. Am J Surg 2019;218:521-6.

9. Ng YY, Ngu JC, Wong AS. Small bowel obstruction in the virgin abdomen: time to challenge surgical dogma with evidence. ANZ J Surg 2018;88:91-4.

10. Zielinski MD, Bannon MP. Current management of small bowel obstruction. Adv Surg 2011;45:1-29.

11. Collom ML, Duane TM, Campbell-Furtick M, Moore BJ, Haddad $\mathrm{NN}$, Zielinski MD, et al. Deconstructing dogma: nonoperative management of small bowel obstruction in the virgin abdomen. J
Trauma Acute Care Surg 2018;85:33-6.

12. Azar T, Berger DL. Adult intussusception. Ann Surg 1997;226: 134-8.

13. Wilson JM, Melvin DB, Gray GF, Thorbjarnarson B. Primary malignancies of the small bowel: a report of 96 cases and review of the literature. Ann Surg 1974;180:175-9.

14. Miller G, Boman J, Shrier I, Gordon PH. Etiology of small bowel obstruction. Am J Surg 2000;180:33-6.

15. Beall DP, Fortman BJ, Lawler BC, Regan F. Imaging bowel obstruction: a comparison between fast magnetic resonance imaging and helical computed tomography. Clin Radiol 2002;57:71924.

16. Zissin R, Hertz M, Paran H, Bernheim J, Shapiro-Feinberg M, Gayer G. Small bowel obstruction secondary to Crohn disease: CT findings. Abdom Imaging 2004;29:320-5.

17. Vailas MG, Moris D, Orfanos S, Vergadis C, Papalampros A. Seatbelt sign in a case of blunt abdominal trauma; what lies beneath it? BMC Surg 2015;15:121.

18. Reisner RM, Cohen JR. Gallstone ileus: a review of 1001 reported cases. Am Surg 1994;60:441-6. 